\title{
Diagnosis of carpal tunnel syndrome
}

A jointly sponsored AAN/AAEM and AAPM \& $\mathrm{R}$ practice parameter reviews the literature through December 2000 and sets standards for electrodiagnostic assessment of CTS. The article also provides concise recommendations for future assessment of diagnostic studies for CTS.

see page 1589

Chang et al. found that wrist-palm motor conduction velocity is more sensitive than wrist-palm sensory conduction velocity in the early diagnosis of CTS, suggesting that sensory fibers are no more susceptible than motor fibers to compression in carpal tunnel. They recommend that in patients with suspected CTS in whom conventional nerve conduction studies are normal, both motor and sensory wrist-palm conduction studies should be carried out to increase the diagnostic yield.

see page 1603

\section{MR for carpal tunnel syndrome}

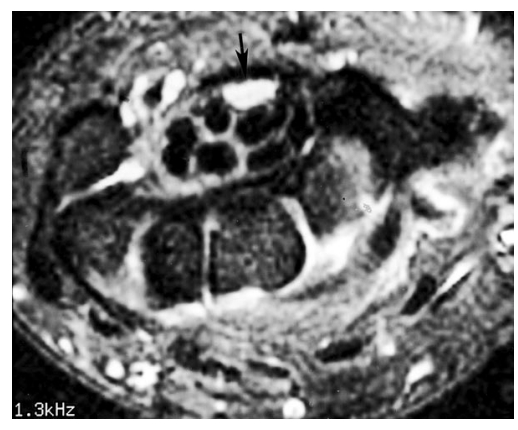

Axial STIR image (swollen and markedly hyperintense median nerve [arrow]).
Jarvik et al. prospectively evaluated the accuracy of high-resolution MR of the carpal tunnel in patients with suspected carpal tunnel syndrome. MR proved highly reliable but only moderately accurate compared to a reference standard that combined electrodiagnostic studies and a hand pain diagram.

see page 1597

The editorial by Fleckenstein and Wolfe accompanying these three papers on CTS considers the relative value of neurophysiologic studies vs MRI for CTS diagnosis. Reviewing clinical settings where MRI will provide valuable data on anatomic abnormalities of peripheral nerve-tumor, arthropathies, developmental anomalies, and following unsuccessful surgery-they conclude that at best MRI falls short of electrodiagnostic assessment of CTS and is more expensive. MRI of peripheral nerve also requires expertise not as widely available as that for electrodiagnostic studies. They also note that the search for a diagnostic test that is 100\% sensitive and 100\% specific should not necessarily be the goal; in mild cases, conservative management is appropriate and spontaneous improvement is possible, so that some degree of diagnostic uncertainty is acceptable.

see page 1583

\section{The prevalence of frontotemporal dementia (FTD)}

Ratnavalli et al. identified/examined 108 cases of early-onset dementia in Cambridgeshire, UK (population 326,019). The age-adjusted prevalence of FTD and $\mathrm{AD}$ in the 45- to 64-year age group was the same (15 per 100,000), suggesting that FTD is a relatively common cause of early-onset dementia.

see page 1615

\section{Accuracy of clinical criteria for FTD: Autopsy assessment}

Rosen et al. compared 30 autopsy-proven FTD cases and $30 \mathrm{AD}$ cases and found that five features best distinguished FTD from AD: social conduct disorders, hyperorality, akinesia, and absence of amnesia and absence of a perceptual disorder. 


\section{CSF tau and Aß42 for diagnosis in patients with FTD}

Riemenschneider et al. studied CSF tau and Aß42 levels in 34 patients with FTD, 74 patients with $\mathrm{AD}$, and 40 controls. These CSF markers differed between FTD and $\mathrm{AD}$, but contributed mainly to negative predictive value in differential diagnosis.

see page 1622

The accompanying editorial on FTD by Galasko and Marder points out that the Ratnavalli et al. study gives the first estimate of the frequency of FTD in the community -and that somewhat surprisingly, it is equal in prevalence to early-onset $A D$. Diagnosis of FTD remains a challenge and although low CSF tau and A 342 levels can serve to make FTD unlikely, no single or combination of diagnostic tests can establish the diagnosis. Clinical criteria are improving as evidenced by the Rosen et al. report. Prospective studies of both brief office screening criteria and more detailed assessments are now needed to further strengthen diagnostic criteria.

see page 1585

\section{Cerebral amyloid angiopathy: Impaired cognitive function}

Cerebral amyloid angiopathy (CAA) is commonly found in neuropathologic investigations of the elderly, but its functional significance is not known. In a population-based autopsy study, Pfeifer et al. found CAA alone was not associated with antemortem cognitive function, but in combination with $\mathrm{AD}$ it was associated with a significant decrement in cognitive function.

see page 1629

The accompanying editorial by Greenberg notes that vascular amyloid, now recognized as the major cause of intracerebral lobar hemorrhage in the elderly, can cause dementia by vascular deposition of amyloid: amyloid distinct from that of AD tissue, but nonetheless, associated with cognitive loss.

see page 1587

\section{Nontyphoidal salmonella encephalopathy}

Arii et al. report eight children (age 1 to 14 years) presenting with seizures or loss of consciousness caused by noninfectious encephalopathy associated with bacteriologically confirmed salmonellosis. There was no evidence of severe dehydration, sepsis, or endotoxemia. This encephalopathy is a distinctive clinical entry that can be differentiated from Reye's syndrome.

see page 1641

\section{Genetic basis of HARP syndrome}

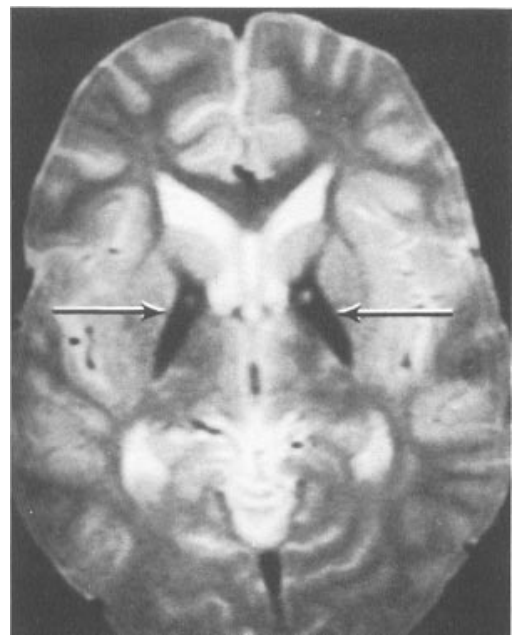

Ching et al. have identified mutations in the gene mutated in pantothenate kinase associated neurodegeneration (PKAN, formerly Havervorden-Spatz syndrome) in the original patient reported in Neurology with hypoprebetalipoproteinemia, acanthocytosis, retinitis pigmentosa, and pallidal degeneration (HARP).

see page 1673 


\section{Neurology}

June 11 Highlights

Neurology 2002;58;1581-1582

This information is current as of June 11, 2002

\section{Updated Information \&} Services

Permissions \& Licensing

Reprints including high resolution figures, can be found at: http://n.neurology.org/content/58/11/1581.full

Information about reproducing this article in parts (figures,tables) or in its entirety can be found online at:

http://www.neurology.org/about/about_the_journal\#permissions

Information about ordering reprints can be found online:

http://n.neurology.org/subscribers/advertise

Neurology ${ }^{\circledR}$ is the official journal of the American Academy of Neurology. Published continuously since 1951, it is now a weekly with 48 issues per year. Copyright . All rights reserved. Print ISSN: 0028-3878.

Online ISSN: 1526-632X.

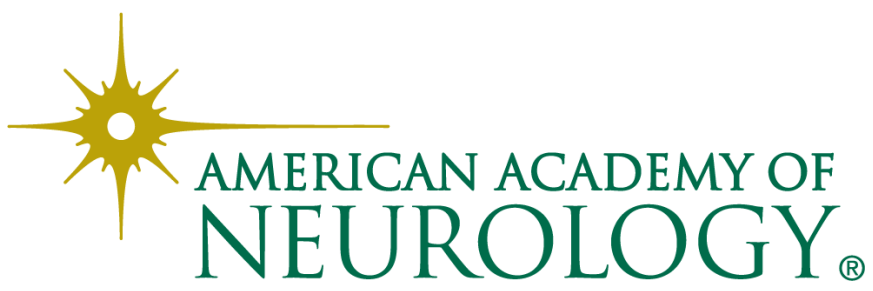

